\title{
Hydration Free Energies of Molecular lons from Theory and Simulation
}

\author{
${ }_{3}$ Maksim Misin, ${ }^{\dagger}$ Maxim V. Fedorov, ${ }^{*}, \dagger$ and David S. Palmer ${ }^{*}$, \\ $4{ }^{\dagger}$ Department of Physics, SUPA, University of Strathclyde, 107 Rottenrow, Glasgow G4 0NG, United Kingdom \\ $5{ }^{\star}$ Department of Pure and Applied Chemistry, University of Strathclyde, 295 Cathedral Street, Glasgow, G1 1XL, United Kingdom \\ 6 S Supporting Information
}

\begin{abstract}
We present a theoretical/computational framework for accurate calculation of hydration free energies of ionized molecular species. The method is based on a molecular theory, 3D-RISM, combined with a recently developed pressure correction (PC+). The 3D-RISM/PC+ model can provide $\sim 3 \mathrm{kcal} / \mathrm{mol}$ hydration free energy accuracy for a large variety of ionic compounds, provided that the Galvani potential of water is taken into account. The results are compared with direct atomistic simulations. Several methodological aspects of hydration free energy calculations for charged species are discussed.
\end{abstract}

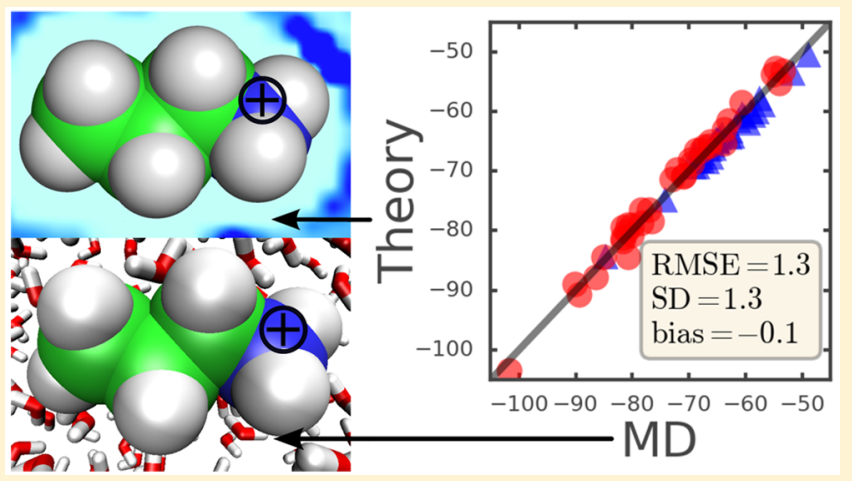

\section{8}

19 Hydration free energy is the most important solvation 20 parameter related to solubility, partitioning of compounds 21 between different phases, and acidity and basicity constants. ${ }^{1-3}$ 22 It is also widely used in the evaluation of ligand binding 23 affinities and in various environmental models. ${ }^{4-6}$

24 While there has been considerable progress in prediction of 25 hydration free energy for neutral compounds, for which various 26 methods can achieve below $1 \mathrm{kcal} / \mathrm{mol}$ accuracy, ${ }^{7-9}$ even the 27 best computational models still show somewhat poor results for 28 charged molecules. ${ }^{7,8}$ We note that research in this direction 29 has been focused on implicit models, which approximate 30 solvent as a dielectric continuum. However, these methods rely 31 on heavy parametrization ${ }^{7,8}$ because they cannot provide 32 information regarding solvent structure and neglect such 33 important effects as solvent polarization, electrostriction, 34 dielectric saturation, etc. ${ }^{10,11}$

35 Explicit solvation models such as molecular dynamics (MD) 36 provide a more realistic view of ionic solvation processes. 37 Unfortunately calculation of solvation free energy is compli38 cated by the need to perform simulations at many intermediate 39 solvation stages. Also, ab initio and even polarizable charge MD 40 is still very computationally expensive, meaning that one has to 41 rely on approximate force fields, which have not been 42 extensively tested nor optimized for ionic compounds. 43 Moreover, for a long time, computation of ionic free energy 44 has presented a substantial theoretical difficulty even with 45 classical MD, mainly due to sensitivity of the obtained energies 46 to the box size and the selected electrostatic method. ${ }^{10,12,13,78}$

47 A group of semiexplicit models, such as three-dimensional 48 reference interaction site model (3D-RISM) or classical density 49 functional theory (CDFT), provide a nice combination of the strengths of both explicit and implicit solvation models. They 50 treat solvent via density correlation functions, capturing many 51 solvation effects which are ignored by implicit models, without 52 the need to simulate a huge number of explicit solvent 53 molecules. ${ }^{1,14,15}$ Both of these models have been used to 54 estimate solvation free energies of atomic ions, but no detailed 55 benchmarks have been published on a series of molecular 56 compounds that are of large interest to bioscience. ${ }^{16,17}$ Here we 57 focus on 3D-RISM, partially because of a recently published 58 pressure correction $(\mathrm{PC}+)$, which significantly improved the 59 accuracy of the model, ${ }^{18,41}$ and partially because this method in 60 recent years has become quite popular and has a readily 61 available AMBER implementation. ${ }^{67}$

This study is split into two parts. First, we compare the 63 results obtained using the 3D-RISM/PC+ model to fixed 64 charge molecular dynamic simulations. We note that charged 65 solutes, unlike neutral ones, contain an additional contribution 66 to solvation free energy that is related to the solvent Galvani 67 potential. ${ }^{19-21}$ We propose a method to estimate it in the 3D- 68 RISM framework and numerically demonstrate its validity. In 69 the second part of the study, we compare classical force field 70 results by the MD and 3D-RISM methods with experimental 71 solvation free energies. In our benchmark we focus on molecular 72 and not atomic ions. We show that even without taking into 73 account effects such as water polarization or charge transfer, it 74 is possible to obtain ionic hydration free energies with 75 reasonable accuracy by employing commonly used force fields. 76

Received: November 4, 2015

Revised: December 15, 2015 


\section{THEORY}

3D-RISM. 3D-RISM allows one to predict the equilibrium 79 distribution of solvent around a solute without explicitly 80 sampling different system configurations. The structure of 81 solvent is described by solute-solvent site correlation 82 functions. The theory is based on the following equation

$$
h_{\alpha 3}(\mathbf{r})=\sum_{\beta=1}^{n_{\mathrm{s}}}\left(\chi_{\alpha \beta}{ }^{*} c_{\beta}\right)(\mathbf{r})
$$

84 where subscripts $\alpha$ and $\beta$ denote indexes of sites in solvent 85 molecule, $n_{\mathrm{s}}$ is the total number of sites in solvent molecule, $h_{\alpha}$ 86 is the total correlation function, $c_{\alpha}$ is the direct correlation 87 function, and the asterisk (*) denotes convolution. ${ }^{1,14} \chi_{\alpha \beta}$ is the 88 solvent susceptibility, typically obtained from 1D-RISM but in 89 principle also obtainable from an $\mathrm{MD}$ or an experimental 90 measurement. ${ }^{1}$ In the hypernetted-chain approximation 91 (HNC), eq 1 is accompanied by the following closure

92

$$
h_{\alpha}(\mathbf{r})+1=\exp \left(-\frac{u_{\alpha}(\mathbf{r})}{k T}+h_{\alpha}(\mathbf{r})-c_{\alpha}(\mathbf{r})\right)
$$

93 where $k$ is the Boltzmann constant, $T$ is temperature, and $u_{\alpha}$ is 94 the potential energy between solute and solvent site $\alpha$, provided 95 as an input. Both eqs 1 and 2 are written for each of the $n_{\mathrm{s}}$ 96 solvent sites. We note that these equations are approximate and 97 do not exactly reproduce solvent distributions obtained from $98 \mathrm{MD}$ simulations, even when tested with the identical force 99 field. $^{1,14}$

100 While conceptually straightforward and thoroughly inves101 tigated, HNC suffers from poor computational convergence. ${ }^{22}$ 102 This problem can be reduced by the use of a partial series 103 expansion of the order $n$ (PSE- $n$ ) of the HNC closure $^{23}$

104

$$
h_{\alpha}(\mathbf{r})= \begin{cases}\sum_{i=0}^{n} \Xi_{\alpha}^{i}(\mathbf{r}) / i !-1 & \text { if } \Xi_{\alpha}(\mathbf{r})>0 \\ \exp \left(\Xi_{\alpha}(\mathbf{r})\right)-1 & \text { if } \Xi_{\alpha}(\mathbf{r}) \leq 0\end{cases}
$$

105 where $\Xi_{\alpha}(\mathbf{r})=-u_{\alpha}(\mathbf{r}) / k T+h_{\alpha}(\mathbf{r})-c_{\alpha}(\mathbf{r})$. Typically larger $n$ 106 values recover HNC results, while smaller $n$ increase the 107 numerical stability of the solution. Choice of $n=3$, denoted as 108 PSE-3, achieves a good balance between convergence and 109 accuracy and has been successfully applied in numerous 110 previous studies. $^{24-26}$

111 Solvation Free Energy. For a solute in solvent, its excess 112 chemical potential $\mu_{\text {sol }}^{\text {ex }}$ is given by Kirkwood's famous formula ${ }^{27}$

113

$$
\mu_{\mathrm{sol}}^{\mathrm{ex}}=\int_{0}^{1} \mathrm{~d} \lambda\left\langle\frac{\partial U(\lambda)}{\partial \lambda}\right\rangle_{\lambda}
$$

114 where $\lambda$ is a solute coupling parameter, $\langle\cdots\rangle_{\lambda}$ indicates an 115 ensemble average at a particular $\lambda$, and $U(\lambda)$ is the potential 116 energy of the solute-solvent system. When a molecule's 117 internal degrees of freedom do not change upon phase transfer, 118 the excess chemical potential is equal to Ben-Naim's definition 119 of solvation free energy, ${ }^{28}$ denoted here as simply $\Delta G$. In this 120 work we assume that this is the case, as we are dealing with 121 primarily small molecules.

122 Equation 4 is in principle exact; direct implementation of this 123 formula in molecular simulations faces difficulties related with 124 numerical convergence and high computational costs for 125 modeling of a large number of intermediate states. Therefore, more efficient ways to evaluate solvation free energy have been 126 developed in both MD- and 3D-RISM-based methods. ${ }^{1,31,78}{ }_{127}$

For $\mathrm{MD}$, one can employ multistate Bennett acceptance ratio 128 (MBAR), a free energy estimation method which has been 129 shown to produce much lower error for the same number of 130 intermediate states between $\lambda=0$ and $\lambda=1$ for practically the 131 same computational cost. ${ }^{29}$ The details of the derivation of the 132 method and its application have been described elsewhere. ${ }^{29,30} 133$

When numerically evaluating $\Delta G_{\mathrm{MD}}$, one typically starts by 134 first decoupling electrostatic energy $U_{\text {Coulomb }}$ and then 135 Lennard-Jones potential $U_{\mathrm{vdW}}{ }^{31}$ On the basis of this scheme 136 one obtains

$$
\Delta G_{\mathrm{MD}}=\Delta G_{\mathrm{Coul}}+\Delta G_{\mathrm{vdW}}
$$

where $\Delta G_{\mathrm{vdW}}$ corresponds to the process of insertion of a 139 molecule with partial charges set to 0 and $\Delta G_{\text {Coul }}$ corresponds 140 to the subsequent charging of the molecule. We note that this 141 equation only holds if intramolecular interactions inside solute 142 do not change during the whole process.

With RISM, one can skip evaluating energies at different 144 values of $\lambda$ and obtain solvation free energy from a single-point 145 calculation. ${ }^{14}$ The exact form of the RISM free energy 146 functional depends on the closure. In the case of $\mathrm{HNC}^{14,32} 147$

$$
\Delta G_{\mathrm{HNC}}=k T \sum_{\alpha=1}^{n_{\mathrm{s}}} \rho_{\alpha} \int_{V}\left[\frac{1}{2} h_{\alpha}^{2}(\mathbf{r})-c_{\alpha}(\mathbf{r})-\frac{1}{2} c_{\alpha}(\mathbf{r}) h_{\alpha}(\mathbf{r})\right]
$$

$\mathrm{d} \mathbf{r}$

(6) 148

For PSE closure

$$
\begin{aligned}
\Delta G_{\mathrm{PSE}-n}= & \mu_{\mathrm{HNC}}^{\mathrm{ex}}-k T \sum_{\alpha=1}^{n_{\mathrm{s}}} \rho_{\alpha} \int_{V}\left[\Theta\left(h_{\alpha}(\mathbf{r})\right)\left(\Xi_{\alpha}(\mathbf{r})\right)^{n+1}\right. \\
& /(n+1) !] \mathrm{d} \mathbf{r}
\end{aligned}
$$

where $\Theta$ is a Heaviside step function. ${ }^{23}$

Functionals presented above are derived from eq 4 and 152 contain no further approximations aside from those involved in 153 3D-RISM derivation. ${ }^{33,34}$ Unfortunately, these expressions are 154 known to yield severely overestimated values for hydration free 155 energies. A number of groups have proposed empirical 156 corrections to $\Delta G_{3 \mathrm{D} \text {-RISM, }}$ but the scope of such corrections 157 remains undetermined.

One of the striking features of both HNC-RISM as well as 159 closely related CDFT in the HRF approximation is largely 160 overestimated solvent pressure, measured in hundreds of 161 atmospheres. $^{25,40}$ Thus, a straightforward correction to over- 162 estimated solvation free energies predicted in both theories may 163 be written as ${ }^{41,42}$

164

$$
\Delta G_{\mathrm{PC}}=\Delta G_{\text {theory }}-P_{\text {theory }} \Delta V+P_{\exp } \Delta V
$$

where PC stands for pressure correction and $P_{\exp }$ is 166 experimental pressure. In $3 \mathrm{D}-\mathrm{RISM} / \mathrm{HNC}^{41}$

$$
P_{\mathrm{HNC}}=\frac{n_{\mathrm{s}}+1}{2} \rho k T-\frac{k T}{2} \rho^{2} \hat{c}(k=0)
$$

where $n_{\mathrm{s}}$ is the number of solvent sites, $\rho$ is the number density 169 of the solvent, and $\hat{c}(k=0)$ is the integral of solvent-solvent 170 direct correlation functions given in site-site formalism by 171

$$
\hat{c}(k=0)=\sum_{\alpha=1}^{n_{s}} \sum_{\alpha^{\prime}=1}^{n_{s}} 4 \pi \int_{0}^{\infty} c_{\alpha \alpha^{\prime}}(r) r^{2} \mathrm{~d} r
$$


173 Alternatively $\hat{c}(k=0)$ can be expressed using the pure solvent 174 isothermal compressibility, $\chi_{\mathrm{T}}$, obtained during evaluation of $175 \chi_{\alpha \beta}$ from 1D-RISM: $\hat{c}(k=0)=1 / \rho-1 /\left(\rho k T \chi_{\mathrm{T}}\right)$. We note that 176 experimental expansion work $P_{\exp } \Delta V$ in eq 8 is not strictly 177 necessarily as at standard conditions this term is very close to 0 . 178 Recent results show that the pressure correction decreases the 179 root-mean-square error of 3D-RISM/HNC predictions from 180 around 16 to $4 \mathrm{kcal} / \mathrm{mol}$ for neutral organic molecules. ${ }^{41}$

181 Interestingly, in both theories it is not the actual PC that is 182 most successful in predicting experimental hydration free 183 energies but a closely related PC+ correction $^{18,41,43,44}$

184

$$
\Delta G_{\mathrm{PC}+}=\Delta G_{\text {theory }}-P_{\text {theory }} \Delta V+P_{\text {ideal }} \Delta V
$$

185 where $P_{\text {ideal }}$ is the ideal gas pressure given by $P_{\text {ideal }}=\rho k T$. Note 186 that due to a misinterpretation, in earlier references ${ }^{18,43,44} \mathrm{PC}+$ 187 was originally called the initial state correction or ISc. 188 Combination of $\mathrm{PC}+$ correction and 3D-RISM/HNC (also 189 PSE-3) approximations was shown to predict hydration free 190 energies of neutral solutes with accuracies of around $1 \mathrm{kcal} /$ $191 \mathrm{~mol}$, which is comparable to MD results with the similar force 192 field.

193 As yet, there is no compelling explanation of why $\mathrm{PC}+$ is 194 more accurate than PC and why it provides significant accuracy 195 improvement for both 3D-RISM and CDFT. In ref 18 we 196 showed that the accuracy of PC+, unlike other approximate 197 models, is independent of temperature. Li et al. also provided 198 arguments in support of PC+ based on asymptotic analysis, ${ }^{44}$ 199 but similar investigation done by Sergiievskyi et al. arrived at a 200 different conclusion. The present work provides further support 201 for PC+ by showing its success in application to charged 202 molecules.

203 The 3D-RISM pressure, taken from ref 41, was derived for 204 HNC closure. However, we believe it can be also used in 205 combination with PSE-3. The difference in numerical 206 predictions of these two closures is minimal, but the good 207 convergence of PSE-3 makes it much more attractive for 208 practical applications. ${ }^{23}$ Therefore, in this paper all actual 209 calculations are performed using PSE-3 closure.

210 Finally, we note that $\Delta G_{\text {Coul }}$ and $\Delta G_{\text {vdW }}$ can also be obtained 211 using 3D-RISM. ${ }^{38}$ Similarly to $\mathrm{MD}, \Delta G_{\mathrm{vdW}}^{\mathrm{BD}-\mathrm{RISM}}$ is the solvation 212 free energy of solute with all partial charges set to 0 . The 213 Coulomb part is then

$214 \Delta G_{3 \mathrm{D}-\mathrm{RISM}}^{\mathrm{Coul}}=\Delta G_{3 \mathrm{D}-\mathrm{RISM}}-\Delta G_{3 \mathrm{D}-\mathrm{RISM}}^{\mathrm{vdW}}$

215 Ionic Solvation Free Energy. The suggested framework 216 for calculation of ionic solvation free energy is largely based on 217 reviews by Hunenberger et al. ${ }^{10}$ and Lin et al. ${ }^{19}$

218 Solvation free energy of a single ion can be defined using two 219 hypothetical processes (Figure 1). In the first process, referred 220 to as a physical process, the ion is reversibly transferred from 221 vacuum to the bulk solvent. During the transfer the ion is 222 moved from a sufficiently large distance from the solvent, 223 through the surface, into the bulk solution. In the second 224 process, referred to as an unphysical process, the ion is slowly 225 "grown" into the solvent. At the initial stage the ion is present 226 only as a ghost particle and does not interact with the solvent. 227 Afterward, the van der Waals interactions between the ion and 228 the system are reversibly switched on. At the final stage the ion 229 is reversibly charged.

230 Two processes share essentially identical initial states (ion 231 which does not interact with the solution is equivalent to ion in 232 vacuum) and final states and thus should correspond to

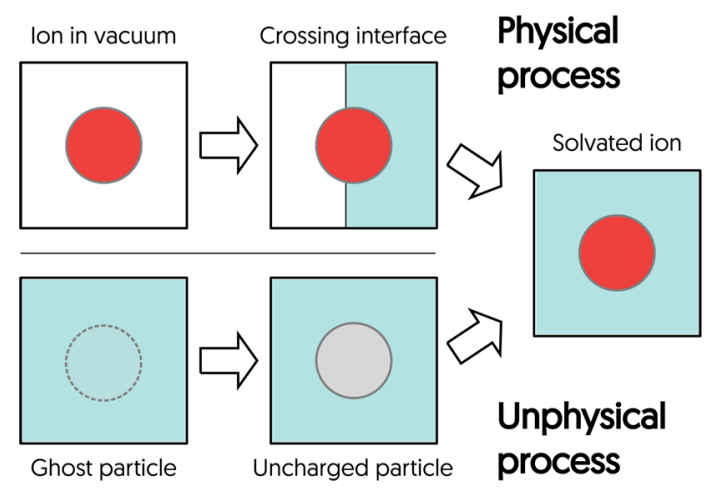

Figure 1. Two hypothetical processes describing solvation free energy of ion. The physical process (shown on top) has (1) ion in vacuum, (2) ion crossing liquid interface, and (3) ion in bulk solvent. The unphysical process (shown on bottom) has (1) ion completely noninteracting with liquid, (2) ion interacting with liquid via van der Waals forces, and (3) ion in bulk solvent. The figure is based on Figure 2.2 from ref 10

identical Gibbs free energy change, which we will refer to as the 233 real solvation free energy $\Delta G_{\text {real }}$. This free energy can be 234 formally decomposed into the bulk and surface contribu- 235 tions ${ }^{10,20,21,45-47}$

$$
\Delta G_{\text {real }}=\Delta G_{\text {intr }}+\Delta G_{\text {surf }}
$$

The bulk contribution $\Delta G_{\text {intr }}$ is called the intrinsic solvation 238 free energy and arises from the interaction between the ion and 239 the local solution environment. The surface term $\Delta G_{\text {surf }}$ arises 240 from the surface polarization, which in turns leads to an 241 electrostatic potential jump of magnitude $\chi$ in air to liquid 242 direction. The contribution of the surface term is purely 243 electrostatic and given by the product of the total charge of the 244 molecule $Q$ and the electrostatic potential difference between 245 the initial and the final positions of the molecule. ${ }^{10}$ When the 246 outside potential (Volta potential) is $0^{19}$

$$
\Delta G_{\text {real }}=\Delta G_{\text {intr }}+\chi Q=\Delta G_{\text {intr }}+\phi_{\mathrm{G}} Q
$$

where $Q$ is the total charge of the ion and $\phi_{\mathrm{G}}$ is the solvent 249 Galvani potential.

Note that physical and unphysical processes result in 251 identical solvation free energies only if the solvent has an 252 explicit surface. If the unphysical process is performed in a 253 system where the whole space is occupied by the solvent, 254 without any boundary between air and liquid, the resulting free 255 energies are intrinsic. Thus, solvation free energies estimated 256 using molecular dynamics with periodic boundary condition 257 and Ewald summation as well as 3D-RISM are, in fact, 258 intrinsic. $^{10,19,48}$

While solvation free energies obtained from simulation are 260 intrinsic, experimental solvation free energies are usually real. ${ }^{10} 261$ In this article we are using experimental ionic free energies from 262 a Minnesota solvation database. ${ }^{49,50}$ These values were 263 obtained by determining solvation free energies of neutral 264

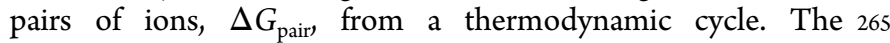
individual hydration free energies were subsequently obtained 266 by combining the single hydration free energy of proton, 267 measured by Tissandier et al. $(265.9 \mathrm{kcal} / \mathrm{mol}),{ }^{, 1}$ with $\Delta G_{\text {pair }} .268$ As follows from eq $14, \Delta G_{\text {pair }}$ can be decomposed into either 269 intrinsic or real solvation free energies 
271

$$
\begin{aligned}
\Delta G_{\text {pair }} & =\Delta G_{\text {real,C }}+\Delta G_{\text {real }, \mathrm{A}} \\
& =\Delta G_{\text {intr, } \mathrm{C}}+\Delta G_{\text {intr, } \mathrm{A}}+0 \times \phi_{\mathrm{G}}
\end{aligned}
$$

272 where subscripts $\mathrm{C}$ and A stand for anion and cation. 273 Ultimately, the question of whether the experimental hydration 274 free energies of ions used in this study are real or intrinsic is 275 related to the question of whether the free energy of hydration 276 of a proton is real or intrinsic. A number of articles suggest that 277 the value by Tissandier et al. is real and does contain a 278 contribution from the surface potential. ${ }^{50,52,53}$

279 Clearly, if we want to compare results obtained from 280 simulations to experimentally measured solvation free energies, 281 we need to make sure that both sets of energies are either real 282 or intrinsic. Due to difficulties associated with measurement of 283 the experimental Galvani potential, ${ }^{10,19}$ the only way we can 284 make such comparison is by evaluating the Galvani potential 285 from simulations and subsequently converting simulation 286 intrinsic free energies to real ones.

287 Other authors have named additional arguments supporting 288 the use of $\Delta G_{\text {real }}$ when discussing simulated results. First, 289 intrinsic solvation free energies depend on the conventional 290 quadrupole moment of the solvent molecule, which is 291 problematic. Lin et al. point out that by embedding an 292 isotropic quadrupole inside the oxygen core one can engineer 293 two water models that will produce identical liquid structure 294 and dynamics but yield arbitrary large differences in $\Delta G_{\text {intr }}$ 295 (they would also have different $\phi_{\mathrm{G}}$ ). ${ }^{19}$ The difference will 296 disappear if one considers $\Delta G_{\text {real }}$ instead. ${ }^{10,21,47,48,52,54}$ In 297 addition, the use of real solvation free energies removes a 298 hotly debated ${ }^{13,55-58}$ difference in the results obtained with P 299 and M summation schemes. ${ }^{10}$ We note that these cancellations 300 only occur if both intrinsic solvation free energies and Galvani 301 potential were evaluated using the same theoretical model.

302 Calculations of Galvani Potential. In this section we 303 discuss ways of evaluating the Galvani potential in both MD 304 and 3D-RISM.

305 In molecular dynamics $\phi_{\mathrm{G}}$ can be obtained by simulating a 306 liquid droplet in an empty box and integrating the Poisson 307 equation

308

$$
\phi_{\mathrm{G}}=\phi\left(z_{\mathrm{w}}\right)-\phi\left(z_{\mathrm{v}}\right)=\int_{z_{\mathrm{v}}}^{z_{\mathrm{w}}} \mathrm{d} z^{\prime} z^{\prime}\left\langle\rho\left(z^{\prime}\right)\right\rangle
$$

309 where $\phi$ is the potential relative to infinity, $z_{\mathrm{w}}$ and $z_{\mathrm{v}}$ are 310 positions deep into liquid and vacuum, respectively, and $311\left\langle\rho\left(x^{\prime}\right)\right\rangle$ is an average charge density at position $z$. For SPC/E 312 water it has been calculated to be $-14.9 \mathrm{kcal} / \mathrm{mol} / \mathrm{e}(-650$ $313 \mathrm{mV}) .^{52}$

314 As simulating the explicit air-water boundary in a standard 315 formulation of 3D-RISM is not possible, a different approach 316 has to be adopted. For an orientationally disordered liquid, the 317 Galvani potential is proportional to the trace of (conventional) 318 quadrupole moment of the solvent molecule (here we are 319 referring to the so-called internal potential). ${ }^{10,19,54}$ For a single320 site solvent, the references cited above provide an analytical 321 formula, but for the solvent with multiple sites, the Galvani 322 potential has to be evaluated numerically by calculating the 323 average electrostatic potential in the center of a small cavity 324 (hard sphere). ${ }^{10,19}$ A small, hard cavity ensures that there is no 325 significant perturbation of liquid near the surface of the cavity 326 and that the only contribution to the potential inside the sphere 327 is due to the quadrupole moment (extensive discussion 328 regarding this can be found in section 4.3 .8 of ref 10 ).
It was shown that this approximation is relatively correct for 329 water at room temperature. ${ }^{13}$ In 3D-RISM the electrostatic 330 potential at a given point can be calculated using ${ }^{59}$

$$
\phi\left(\mathbf{r}_{0}\right)=\frac{1}{4 \pi \epsilon_{0}} \sum_{\alpha=1}^{n_{\mathrm{s}}} \int_{V} \frac{g_{\alpha}(\mathbf{r}) \rho_{\alpha} q_{\alpha}}{r} \mathrm{~d} \mathbf{r}
$$

where $r$ is the distance from $\mathbf{r}_{\mathbf{0}}$ to $\mathbf{r}, \epsilon_{0}$ is the permittivity of 333 vacuum, $g_{\alpha}(r)=h_{\alpha}(r)+1, \rho_{\alpha}$ is the number density of the site 334 $\alpha$, and $q_{\alpha}$ is the partial charge of the site $\alpha$. The Galvani 335 potential of the solvent was estimated by calculating $\phi\left(\mathbf{r}_{\mathbf{0}}\right)$ in 336 the center of the cavity. Using the radius of the cavity equal to 337 $0.5 \AA$ (same as grid size), we estimated $\phi_{\mathrm{G}}$ to be $-13.43 \mathrm{kcal} / 338$ $\mathrm{mol} / \mathrm{e}$ for $\mathrm{cSPC} / \mathrm{e}$ water and $-12.55 \mathrm{kcal} / \mathrm{mol} / \mathrm{e}$ for cTIP3P 339 water.

340

Finite-Size Corrections. 3D-RISM is implemented on a 341 nonperiodic grid and, therefore, corresponds to an infinitely 342 diluted solute. ${ }^{60}$ However, in PBC/Ewald MD simulations a 343 solvated ion interacts with its periodic images; this introduces a 344 number of periodicity related artifacts (finite-size ef- 345 fects). ${ }^{12,13,61-63}$ For single atomic ions there are analytical 346 formulas which let one correct free energies resulting from MD 347 simulation to reflect solvation in a nonperiodic system. ${ }^{10,13}$ For 348 polyatomic ions there are no analytical formulas and one has to 349 run two Poisson-Boltzmann simulations for periodic and 350 nonperiodic systems to quantify the energetic effect of 351 periodicity. $^{63,64}$

Fortunately, the magnitude of most of these errors scales as 353 the ratio of the molecule size to the box length and becomes 354 unimportant in the large box limit. A way to correct the finite- 355 size errors without resorting to periodic Poisson-Boltzmann 356 calculations has been suggested in the paper by Rocklin et al. ${ }^{63} 357$

$$
\Delta \Delta G_{\mathrm{ANA}}=\Delta \Delta G_{\mathrm{NET}}+\Delta \Delta G_{\mathrm{USV}}+\Delta \Delta G_{\mathrm{RIP}}+\Delta \Delta G_{\mathrm{EMP}}
$$

where $\Delta \Delta G_{\mathrm{ANA}}$ stands for analytical corrections, $\Delta \Delta G_{\mathrm{NET}} 359$ accounts for periodicity-induced net charge interaction, 360 $\Delta \Delta G_{\mathrm{USV}}$ is for periodicity-induced undersolvation, and 361 $\Delta \Delta G_{\mathrm{RIP}}$ is for residual integrated potential effects. $\Delta \Delta G_{\mathrm{EMP}}$ is 362 an empirical term. Out of all these corrections only two scale as 363 an inverse length of the box $L: \Delta \Delta G_{\mathrm{NET}}$ and $\Delta \Delta G_{\mathrm{USV}}$. All other 364 correction terms either decrease rapidly with increasing box size 365 or become significant only for situations involving binding of 366 charged species. Note that there is no need for us to correct the 367 error associated with $\mathrm{P}$ summation since we are combining 368 intrinsic free energies with $\phi_{\mathrm{G}}$, which leads to cancellation of 369 this error. ${ }^{10}$

As we used a large box, only $\Delta \Delta G_{\mathrm{NET}}$ and $\Delta \Delta G_{\mathrm{USV}}$ were 371 taken into account. All ionic hydration free energies obtained 372 from molecular dynamics simulations were corrected as ${ }^{63}$

$$
\begin{aligned}
\Delta G_{\mathrm{MD}, \mathrm{cor}} & =\Delta G_{\mathrm{MD}}+\Delta \Delta G_{\mathrm{USV}}+\Delta \Delta G_{\mathrm{NET}} \\
& =\Delta G_{\mathrm{MD}}-\frac{\xi}{8 \pi \epsilon_{0} \epsilon_{\mathrm{w}}} \frac{Q^{2}}{L}
\end{aligned}
$$

where $\xi$ is the Wigner integration constant for cubic boxes $(\approx 375$ $-2.837297){ }^{65} \epsilon_{\mathrm{w}}$ is the permittivity of model water (73.5 for 376 SPC/E), and $Q$ is the total charge of molecule. A similar 377 shortened correction scheme has been used in other recent 378 publications. ${ }^{19,66}$ We note that with the settings which were 379 used in our study ( $3 \mathrm{~nm}$ sized box), the final finite-size 380 correction is quite small, around $0.2 \mathrm{kcal} / \mathrm{mol}$, and much 381 
382 smaller than the contribution due to water-vacuum potential 383 jump.

\section{METHODOLOGY}

385 Experimental hydration free energies of ionic compounds and 386 their geometries were taken from the 2012 version of the 387 Minnesota solvation database. ${ }^{49,50}$ These values are based on 388 the hydration free energy of a proton $=265.9 \mathrm{kcal} / \mathrm{mol}{ }^{51}$ 389 When selecting compounds from the database we avoided 390 water clusters as well as ions that were structurally similar to 391 other chosen molecules. We ended up selecting 70 compounds 392 in total: 36 anions and 34 cations.

393 For the ionic data set, we assigned GAFF force field 394 parameters and AM1-BCC charges using various programs 395 from the AmberTools 14 package. ${ }^{67}$ We also tested the 396 performance of OPLS-2005 ${ }^{68}$ Lennard-Jones parameters 397 combined with Charge Model 5 partial charges (CM5). ${ }^{69}$ 398 Assignment of OPLS-2005 parameters was performed 399 automatically using Maestro. ${ }^{70}$ For all calculations we used 400 Lorentz-Berthelot mixing rules. ${ }^{71}$ Evaluation of CM5 charges 401 required additional quantum mechanical calculations, which 402 were performed with Gaussian 09, Revision D.01, ${ }^{72}$ using the 403 M06-2X functional ${ }^{73}$ and MG3S basis set. ${ }^{74}$ Solvent was 404 represented using the SMD model. ${ }^{7}$ Charges were extracted 405 from output files using CM5PAC program. ${ }^{75}$

406 Molecular dynamics simulations with ionic compounds were 407 performed using Gromacs 5.04. ${ }^{76}$ We used a cubic box with 408 periodic boundary conditions. The water was represented using 409 a rigid SPC/E model. ${ }^{77}$ In all simulations the system consisted 410 of a single ion and 1024 water molecules; no counterions were 411 used. All bonds with hydrogens were kept rigid using the 412 LINCS algorithm of 12th order. Dynamics was simulated using 413 the Langevin integrator, with a reference temperature of 298.15 $414 \mathrm{~K}$ and a friction constant of $1.0 \mathrm{ps}^{-1}$.

415 For short-range interactions a pair list was generated using a 416 Verlet cutoff scheme. Lennard-Jones interactions were 417 smoothly switched off between 9 and $12 \AA$. The cutoff artifacts 418 were accounted for using long-range pressure and dispersion 419 corrections as implemented in Gromacs. Electrostatics 420 interactions were treated using the particle-mesh Ewald 421 (PME) method $^{79}$ with a $12 \AA$ real space cutoff, $1.2 \AA$ Fourier 422 spacing, 6th order spline interpolation, and tolerance set to $42310^{-6}$

424 To compute the hydration free energy using MD, we 425 performed 20 separate calculations at each $\lambda$, decoupling first 426 electrostatics and then Lennard-Jones interactions between 427 solute and solvent. Intramolecular interactions within solute 428 were kept the same at all lambda values. Calculations with 429 modified electrostatics interactions were performed at $\lambda=0$, $4300.25,0.5,0.75$, and 1. Decoupling of the Lennard-Jones 431 interactions was done using calculations at $\lambda=0,0.05,0.1,0.2$, $4320.3,0.4,0.5,0.6,0.65,0.7,0.75,0.8,0.85,0.9,0.95$, and 1.0.

433 Prior to running $\mathrm{MD}$ simulation at each $\lambda$, we performed 4345000 steps of steepest descent optimization. After that we 435 performed a 200 ps equilibration and 1300 ps production run. 436 The time step was set to 1 fs. In both equilibration and 437 production runs, pressure was kept constant at 1 bar using a 438 Berendsen barosta, ${ }^{80}$ with time constant set to 1 ps and 439 compressibility to $4.5 \times 10^{-5} \mathrm{bar}^{-1}$.

440 After completing the simulations, the intrinsic hydration free 441 energy was evaluated using the Multistate Bennett Acceptance 442 Ratio (MBAR). ${ }^{29}$ The actual calculation was performed using a 443 python script alchemical-analysis.py. ${ }^{31}$
RISM calculations were performed with SPC/E and TIP3P 444 water models that have Lennard-Jones parameters on 445 hydrogen to improve convergence (cSPC/E and cTIP3P). ${ }^{81} 446$ Water susceptibility functions were generated using dielectri- 447 cally consistent 1D-RISM (DRISM) ${ }^{82}$ implemented in the 448 AmberTools 14 package. Water density was set to $997 \mathrm{~kg} / \mathrm{m}^{3} 449$ and dielectric constant to 78.4 (experimental values at $298 \mathrm{~K}$ ). 450 The DRISM equations were solved with tolerance set to $1 \times 451$ $10^{-12}$ and grid spacing to $0.025 \AA$.

452

$3 \mathrm{D}$-RISM calculations were performed using the 453 rism3d.snglpnt program from the AmberTools 14 pack- 454 age. $^{22,81,83}$ The grid spacing was set to $0.5 \AA$, buffer to $25 \AA$, 455 and tolerance to $1 \times 10^{-5}$. While these parameters provide 456 slightly less accurate results compared to the ones used in our 457 previous work, ${ }^{18}$ the resulting errors in obtained hydration free 458 energies are only around $0.1 \mathrm{kcal} / \mathrm{mol}$. On the other hand, they 459 led to a decrease in calculation time from minutes to seconds, 460 which in our opinion is a favorable trade-off. Both 1D-RISM 461 and 3D-RISM calculations were performed using the PSE-3 462 closure. Similarly to our previous work, calculation setup was 463 automated using a Python script available at https://github. 464 com/MTS-Strathclyde/PC_plus.

RESULTS AND DISCUSSION 466

PC+ Model Verification. Figure 2 compares insertion, $467 \mathrm{f} 2$ charging, and hydration free energies from 3D-RISM/PSE-3/ 468
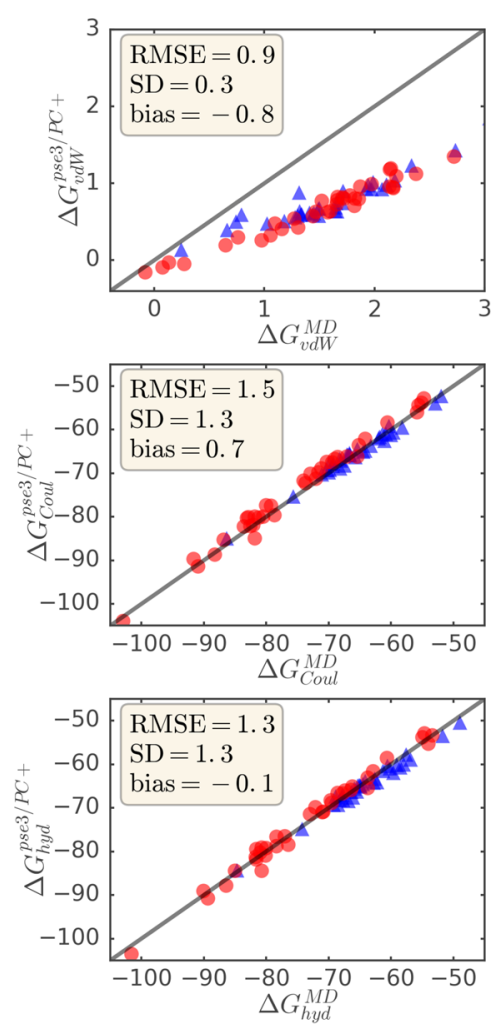

Figure 2. Comparison of predicted insertion free energies (top), charging free energies (middle), and hydration free energies (bottom) of ionic compounds from MD and PSE-3/PC+. In both models, solutes were described using GAFF parameters and AM1-BCC charges. Anions are shown as red circles and cations as blue triangles. All energies are in $\mathrm{kcal} / \mathrm{mol}$. The box in the top left corner of each figure shows root-mean-square error (RMSE), standard deviation (SD), and bias of PSE-3/PC+ results compared to MD. 
$469 \mathrm{PC}+$ (from here on simply $\mathrm{PC}+$ ) and MD/MBAR (or simply $470 \mathrm{MD}$ ), both obtained using GAFF parameters, AM1-BCC 471 charges, and SPC/E water models (cSPC/E for 3D-RISM). 472 Both charging and hydration energies include the water473 vacuum potential as well as finite-size corrections for $\mathrm{MD}$ 474 results. The $\mathrm{PC}+$ model underestimates insertion free energies 475 with respect to $\mathrm{MD}$, but the errors are systematic and 476 proportional to the magnitude of the energy, unlike errors 477 from the PSE-3 closure alone that are more or less random and 478 are much larger in magnitude (uncorrected results are included 479 in the Supporting Information). On the other hand, the 480 agreement between charging free energies from both models is 481 good, with errors of about $1.3 \mathrm{kcal} / \mathrm{mol}$. Since the charging free 482 energies are much larger in magnitude than insertion free 483 energies, hydration free energies are in close agreement. Very 484 similar results, but for neutral compounds, have also been 485 obtained by Kleine et al. ${ }^{84}$

486 Importantly, these results also justify our choice of 487 approximating $\phi_{\mathrm{G}}$ for 3D-RISM. Uncorrected results, which 488 can be found in the Supporting Information, consistently 489 overestimate the hydration free energy of anions and 490 underestimate energies of cations compared to MD by about $4912 \mathrm{kcal} / \mathrm{mol}$. Corrected results still contain charge-dependent 492 bias, but it is much smaller: around $0.4 \mathrm{kcal} / \mathrm{mol}$.

493 Both 3D-RISM and MD free energies have associated 494 uncertainties, but with the parameters we used they are quite 495 small: about $0.08 \mathrm{kcal} / \mathrm{mol}$ on average for $\mathrm{MD}$ and less than $4960.01 \mathrm{kcal} / \mathrm{mol}$ for 3D-RISM. These uncertainties are much 497 smaller than errors associated with models and can be safely 498 ignored for the purposes of this study.

499 The extent of agreement of $\mathrm{PC}+$ results with MD is quite 500 remarkable, considering that for ionic compounds energies are 501 much larger in magnitude compared to neutral compounds. 502 The observed differences can be attributed to the fact that 3D503 RISM uses approximate closure, assumes additivity of direct 504 correlation functions, omits third and higher order correlation 505 functions, and does not sample solute conformations (this 506 problem, however, can be solved by MD/3D-RISM ${ }^{85}$ ). Overall, 507 these results show that PSE-3/PC+ can serve as a good 508 approximation to the much more expensive $\mathrm{MD}$ method: 509 hydration free energies with 3D-RISM using parameters used in 510 this study are evaluated in seconds on a single CPU, compared 511 to approximately $6 \mathrm{~h}$ on 12 CPUs needed to obtain a single free 512 energy from MD. This equates to about 4 orders of magnitude 513 difference in computational power in favor of RISM.

514 Comparison with Experiment. Figure 3 shows a 515 comparison between predicted and experimental solvation 516 free energies. The two top graphs compare results from $\mathrm{MD}$ 517 and PSE-3/PC+ obtained with GAFF/AM1-BCC parameters 518 to experiment. The difference of errors from PSE-3/PC+ and $519 \mathrm{MD}$ is also much smaller than the difference between the 520 models themselves. Thus, for a number of molecules PSE-3/PC $521+$ predicts hydration free energies more accurately than 522 molecular dynamics. This might seem surprising at first, 523 considering that 3D-RISM contains additional sources of errors 524 due to a number of approximations. However, it has been 525 shown that extra Lennard-Jones parameters on hydrogen in 526 the cSPC/E model partially take into account polarization, ${ }^{81}$ 527 which might compensate for additional errors due to the model. 528 The accuracy of the results above is surprising and indicates 529 that hydration free energies obtained with classical point charge 530 models can be relatively reliable even without taking into 531 account polarization, charge transfer, and an accurate
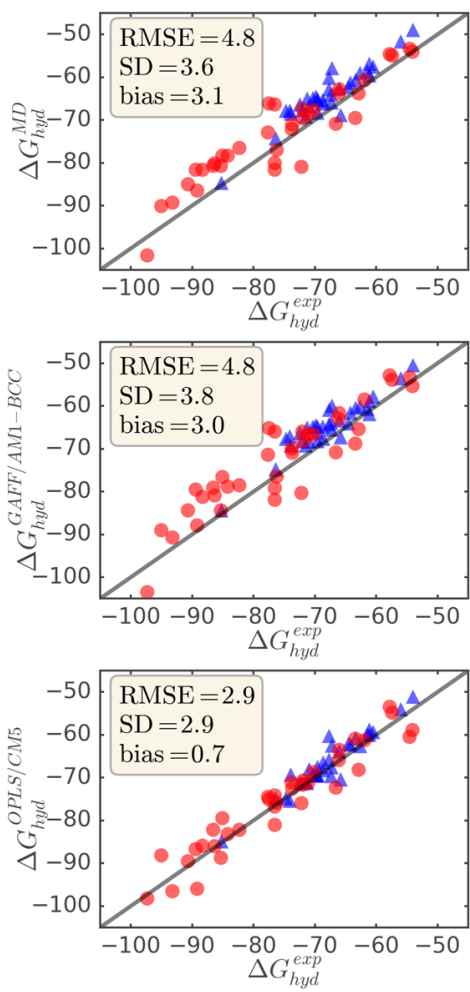

Figure 3. Comparison of hydration free energies of ionic compounds against experiment. (Top and middle) Results by MD and PSE-3/PC +, obtained using GAFF/AM1-BCC parameters. (Bottom) Results from PSE-3/PC+, obtained with OPLS/CM5 parameters.

representation of dispersion interactions. Moreover, they 532 further support the use of "real" hydration free energy as 533 opposed to intrinsic (uncorrected results are provided in the 534 Supporting Information and show clear biases for anions and 535 cations).

536

To test whether we can improve our estimates using different 537 Lennard-Jones parameters and partial charges, we repeated the 538 3D-RISM calculations using combinations of the OPLS-2005 539 force field Lennard-Jones parameters and CM5 charges. We 540 also reran all 3D-RISM calculations with cTIP3P water model, 541 which was intended as a test for both PC+ and Galvani 542 corrections. These results are shown in Table 1 , where we $543 \mathrm{tl}$ additionally presented errors for cations and anions separately. 544

On the basis of the results in Table 1, we see that OPLS/ 545 CM5 parameters outperform GAFF/AM1-BCC for both 546 cSPC/E and CTIP3P water; individual data points obtained 547 with these parameters and cSPC/E water are shown on the 548 bottom graph of Figure 3. For both sets of nonbonded 549 parameters cTIP3P results are slightly worse than cSPC/E. It is 550 interesting that all models overestimate hydration free energy 551 compared to experiment, similarly to the results obtained with 552 the SMD model.?

Compared to GAFF/AM1-BCC, OPLS/CM5 lowers both 554 random error and bias, which is expected considering that this 555 model was parametrized in order to accurately reproduce the 556 electric field of a molecule and uses a more sophisticated 557 electronic structure method to do it. This suggests that the use 558 of QM/3D-RISM that has been proposed and implemented by 559 a couple of other groups might lead to even better results. ${ }^{86,87} 560$

To our knowledge the approach presented here is one of the 561 most accurate ways of predicting ionic hydration free energies 562 through computational models. The RMSE of about $3 \mathrm{kcal} / \mathrm{mol} 563$ 
Table 1. Comparison of Predicted Hydration Free Energies with Experimental Data (kcal/mol)

\begin{tabular}{|c|c|c|c|c|c|c|c|c|c|c|c|}
\hline \multirow[b]{2}{*}{ method } & \multirow[b]{2}{*}{ force field } & \multirow[b]{2}{*}{ water model } & \multicolumn{3}{|c|}{ combined } & \multicolumn{3}{|c|}{ cations } & \multicolumn{3}{|c|}{ anions } \\
\hline & & & RMSE & SD & bias & RMSE & SD & bias & RMSE & SD & bias \\
\hline MD & GAFF/AM1-BCC & SPC/E & 4.76 & 3.61 & 3.13 & 4.43 & 2.28 & 3.82 & 5.05 & 4.46 & 2.47 \\
\hline $\mathrm{PC}+$ & GAFF/AM1-BCC & $\mathrm{cSPC} / \mathrm{E}$ & 4.84 & 3.79 & 3.04 & 3.93 & 2.24 & 3.25 & 5.56 & 4.84 & 2.85 \\
\hline $\mathrm{PC}+$ & OPLS/CM5 & $\mathrm{cSPC} / \mathrm{E}$ & 2.94 & 2.87 & 0.73 & 2.41 & 2.07 & 1.27 & 3.37 & 3.41 & 0.22 \\
\hline $\mathrm{PC}+$ & GAFF/AM1-BCC & сTIP3Р & 5.40 & 3.85 & 3.82 & 3.50 & 2.19 & 2.76 & 6.72 & 4.75 & 4.82 \\
\hline $\mathrm{PC}+$ & OPLS/CM5 & сTIP3Р & 3.20 & 2.87 & 1.45 & 2.28 & 2.07 & 1.02 & 3.87 & 3.44 & 1.86 \\
\hline
\end{tabular}

564 obtained with OPLS/CM5 parameters and the cSPC/E water 565 model is comparable to the $3.25 \mathrm{kcal} / \mathrm{mol}$ mean unsigned error 566 reported for the SMVLE model, albeit on a larger data set. ${ }^{8}$ The 567 approach also outperforms other popular models such as $\mathrm{SMD}^{7}$ 568 and SM8. ${ }^{88}$ Importantly, the results presented here are 569 obtained with force fields, which have not been parametrized 570 for ionic solvation free energies. Therefore, they can be further 571 improved by optimizing the force field parameters that is the 572 subject of our ongoing research.

\section{CONCLUSION}

574 We proposed a new method for estimating the Galvani 575 potential using 3D-RISM. The obtained estimate is then used 576 to compute real hydration free energies of molecular ions with 577 3D-RISM/PSE-3/PC+ models. Our values are in close 578 agreement with both results of direct simulation by force 579 field-based MD and with real experimental free energies. We 580 found that the major source of errors in our calculations was 581 related to the force field parameters and atomic partial charges 582 and not to the approximations used in the 3D-RISM model. 583 The use of OPLS-2005 Lennard-Jones parameters and CM5 584 partial charges substantially decreased disagreement between 585 the 3D-RISM hydration free energies and experiment, bringing 586 the root-mean-square error on a set of 36 anions and 34 cations 587 to about $3 \mathrm{kcal} / \mathrm{mol}$. This level of accuracy makes the method 588 proposed here competitive with the state of the art $\mathrm{MD}$ and 589 continuum solvation approaches. ${ }^{8,88}$

590 This study, together with our previous results for neutral 591 compounds, ${ }^{18}$ shows that 3D-RISM, combined with PSE-3 592 closure and PC+ correction, is a powerful method for 593 predicting thermodynamic parameters of molecular solvation. 594 The results suggest that at least for ionic compounds the 595 accuracy of the 3D-RISM/PCE-3/PC+ model is more limited 596 by the accuracy of the force fields than by the model 597 approximations. A better parametrization of the force fields 598 (potentially using fast 3D-RISM calculations for high599 throughput optimization of parameters) should further reduce 600 the observed errors. The study also revealed a problem related 601 to the PC+ correction-systematic underestimation of the vdW 602 component of the hydration free energy; we plan to address 603 this problem in future articles.

\section{$604 \quad$ ASSOCIATED CONTENT}

\section{S Supporting Information}

606 The Supporting Information is available free of charge on the 607 ACS Publications website at DOI: 10.1021/acs.jpcb.5b10809.

608 Tables with experimental and computed hydration free 609 energies for both data sets (csv); pdb structures of all 610 molecules used in the calculations (zip); results obtained 611 without taking into account Galvani potential or PC+ 612 correction are also presented (pdf).

\section{AUTHOR INFORMATION}

\section{Corresponding Authors}

*E-mail: maxim.fedorov@strath.ac.uk.

*E-mail: david.palmer@strath.ac.uk.

Notes

The authors declare no competing financial interest.

\section{ACKNOWLEDGMENTS}

Results were obtained using the EPSRC funded ARCHIE-WeSt 620 High Performance Computer (www.archie-west.ac.uk. EPSRC 621 grant no. EP/K000586/1). D.S.P. is grateful for funding from 622 the European Commission through a Marie Curie Intra- 623 European Fellowship within the Seventh European Community 624 Framework Programme (FP7-PEOPLE-2010-IEF). M.V.F. 625 thanks the Scottish Universities Physics Alliance (SUPA) for 626 funding through MVFs SUPA2 start-up funds. D.S.P. thanks 627 the University of Strathclyde for support through its Strategic 628 Appointment and Investment Scheme.

\section{REFERENCES}

(1) Ratkova, E. L.; Palmer, D. S.; Fedorov, M. V. Solvation 631 Thermodynamics of Organic Molecules by the Molecular Integral 632 Equation Theory: Approaching Chemical Accuracy. Chem. Rev. 2015, 633 115, 6312-6356.

634

(2) Palmer, D. S.; Llinàs, A.; Morao, I.; Day, G. M.; Goodman, J. M.; 635 Glen, R. C.; Mitchell, J. B. O. Predicting Intrinsic Aqueous Solubility 636 by a Thermodynamic Cycle. Mol. Pharmaceutics 2008, 5, 266-279. 637

(3) Alongi, K. S.; Shields, G. C. In Annual Reports in Computational 638 Chemistry; Wheeler, R. A., Ed.; Elsevier: New York, 2010; Vol. 6, pp 639 113-138.

(4) Gilson, M. K.; Zhou, H.-X. Calculation of Protein-Ligand Binding 641 Affinities. Annu. Rev. Biophys. Biomol. Struct. 2007, 36, 21-42. 642

(5) Ratkova, E. L.; Fedorov, M. V. Combination of RISM and 643 Cheminformatics for Efficient Predictions of Hydration Free Energy of 644 Polyfragment Molecules: Application to a Set of Organic Pollutants. J. 645 Chem. Theory Comput. 2011, 7, 1450-1457.

(6) Takeuchi, M.; Matubayasi, N.; Kameda, Y.; Minofar, B.; Ishiguro, 647 S.-i.; Umebayashi, Y. Free-Energy and Structural Analysis of Ion 648 Solvation and Contact Ion-Pair Formation of $\mathrm{Li}+$ with BF4- and 649 PF6- in Water and Carbonate Solvents. J. Phys. Chem. B 2012, 116, 650 6476-6487.

(7) Marenich, A. V.; Cramer, C. J.; Truhlar, D. G. Universal Solvation 652 Model Based on Solute Electron Density and on a Continuum Model 653 of the Solvent Defined by the Bulk Dielectric Constant and Atomic 654 Surface Tensions. J. Phys. Chem. B 2009, 113, 6378-6396.

(8) Liu, J.; Kelly, C. P.; Goren, A. C.; Marenich, A. V.; Cramer, C. J.; 656 Truhlar, D. G.; Zhan, C.-G. Free Energies of Solvation with Surface, 657 Volume, and Local Electrostatic Effects and Atomic Surface Tensions 658 to Represent the First Solvation Shell. J. Chem. Theory Comput. 2010, 659 6, 1109-1117.

(9) Mobley, D. L.; Bayly, C. I.; Cooper, M. D.; Shirts, M. R.; Dill, K. 661 A. Small Molecule Hydration Free Energies in Explicit Solvent: An 662 Extensive Test of Fixed-Charge Atomistic Simulations. J. Chem. Theory 663 Comput. 2009, 5, 350-358. 
665 (10) Hunenberger, P.; Reif, M. Single-Ion Solvation: Experimental and 666 Theoretical Approaches to Elusive Thermodynamic Quantities; Royal 667 Society of Chemistry: Cambridge, 2011.

668 (11) Harris, R. C.; Pettitt, B. M. Examining the Assumptions 669 Underlying Continuum-Solvent Models. J. Chem. Theory Comput. 670 2015, 11, 4593-4600.

671 (12) Kastenholz, M. A.; Hünenberger, P. H. Computation of 672 Methodology-Independent Ionic Solvation Free Energies from 673 Molecular Simulations. II. The Hydration Free Energy of the Sodium 674 Cation. J. Chem. Phys. 2006, 124, 224501.

675 (13) Kastenholz, M. A.; Hünenberger, P. H. Computation of 676 Methodology-Independent Ionic Solvation Free Energies from 677 Molecular Simulations. I. The Electrostatic Potential in Molecular 678 Liquids. J. Chem. Phys. 2006, 124, 124106.

679 (14) Hirata, F. Molecular Theory of Solvation; Kluwer Academic 680 Publishers: New York, 2003.

681 (15) Jeanmairet, G.; Levesque, M.; Sergiievskyi, V.; Borgis, D. 682 Computational Trends in Solvation and Transport in Liquids; Schriften 683 des Forschungszentrums Jülich: Jülich, 2015; Vol. 28, pp 187-208.

684 (16) Zhao, S.; Jin, Z.; Wu, J. New Theoretical Method for Rapid 685 Prediction of Solvation Free Energy in Water. J. Phys. Chem. B 2011, 686 115, 6971-6975.

687 (17) Chiodo, S.; Chuev, G. N.; Erofeeva, S. E.; Fedorov, M. V.; 688 Russo, N.; Sicilia, E. Comparative Study of Electrostatic Solvent 689 Response by RISM and PCM Methods. Int. J. Quantum Chem. 2007, 690 107, 265-274.

691 (18) Misin, M.; Fedorov, M. V.; Palmer, D. S. Accurate Hydration 692 Free Energies at a Wide Range of Temperatures from 3D-RISM. J. 693 Chem. Phys. 2015, 142, 091105.

694 (19) Lin, Y.-L.; Aleksandrov, A.; Simonson, T.; Roux, B. An Overview 695 of Electrostatic Free Energy Computations for Solutions and Proteins. 696 J. Chem. Theory Comput. 2014, 10, 2690-2709.

697 (20) Horváth, L.; Beu, T.; Manghi, M.; Palmeri, J. The Vapor-Liquid 698 Interface Potential of (Multi)Polar Fluids and its Influence on Ion 699 Solvation. J. Chem. Phys. 2013, 138, 154702.

700 (21) Remsing, R. C.; Baer, M. D.; Schenter, G. K.; Mundy, C. J.; 701 Weeks, J. D. The Role of Broken Symmetry in Solvation of a Spherical 702 Cavity in Classical and Quantum Water Models. J. Phys. Chem. Lett. 703 2014, 5, 2767-2774.

704 (22) Kovalenko, A.; Hirata, F. Self-Consistent Description of a Metal705 Water Interface by the Kohn-Sham Density Functional Theory and the 706 Three-Dimensional Reference Interaction Site Model. J. Chem. Phys. 707 1999, 110, 10095-10112.

708 (23) Kast, S. M.; Kloss, T. Closed-Form Expressions of the Chemical 709 Potential for Integral Equation Closures with Certain Bridge 710 Functions. J. Chem. Phys. 2008, 129, 236101.

711 (24) Luchko, T.; Joung, I. S.; Case, D. A. Innovations in Biomolecular 712 Modeling and Simulations; RSC Publishing: London, UK, 2012; Vol. 1. 713 (25) Joung, I. S.; Luchko, T.; Case, D. A. Simple Electrolyte 714 Solutions: Comparison of DRISM and Molecular Dynamics Results 715 for Alkali Halide Solutions. J. Chem. Phys. 2013, 138 (4), 044103.

716 (26) Giambaşu, G. M.; Luchko, T.; Herschlag, D.; York, D. M.; Case, 717 D. A. Ion Counting from Explicit-Solvent Simulations and 3D-RISM. 718 Biophys. J. 2014, 106, 883-894. 00009

719 (27) Kirkwood, J. G. Statistical Mechanics of Fluid Mixtures. J. Chem. 720 Phys. 1935, 3, 300-313.

721 (28) Ben-Naim, A. Molecular Theory of Solutions; OUP: Oxford, 2006. 722 (29) Shirts, M. R.; Chodera, J. D. Statistically Optimal Analysis of 723 Samples from Multiple Equilibrium States. J. Chem. Phys. 2008, 129, 724124105.

725 (30) Mobley, D. L.; Liu, S.; Cerutti, D. S.; Swope, W. C.; Rice, J. E. 726 Alchemical Prediction of Hydration Free Energies for SAMPL. J. 727 Comput.-Aided Mol. Des. 2012, 26, 551-562.

728 (31) Klimovich, P. V.; Shirts, M. R.; Mobley, D. L. Guidelines for the 729 Analysis of Free Energy Calculations. J. Comput.-Aided Mol. Des. 2015, 730 29, 397-411.

731 (32) Sergievskii, V. P. Modelling of Solvation Thermodynamics by 732 Using a Combination of Reference Interaction Site Model Theory and
Multi-grid Numerical Methods. Doctoral thesis, University of 733 Strathclyde, Glasgow, 2013.

(33) Beglov, D.; Roux, B. Solvation of Complex Molecules in a Polar 735 Liquid: An Integral Equation Theory. J. Chem. Phys. 1996, 104, 8678- 736 8689.

(34) Cortis, C. M.; Rossky, P. J.; Friesner, R. A. A Three-Dimensional 738 Reduction of the Ornstein-Zernicke Equation for Molecular Liquids. J. 739 Chem. Phys. 1997, 107, 6400-6414.

(35) Palmer, D. S.; Frolov, A. I.; Ratkova, E. L.; Fedorov, M. V. 741 Towards a Universal Method for Calculating Hydration Free Energies: 742 a 3D Reference Interaction Site Model with Partial Molar Volume 743 Correction. J. Phys.: Condens. Matter 2010, 22, 492101.

(36) Ratkova, E. L.; Chuev, G. N.; Sergiievskyi, V. P.; Fedorov, M. V. 745 An Accurate Prediction of Hydration Free Energies by Combination of 746 Molecular Integral Equations Theory with Structural Descriptors. J. 747 Phys. Chem. B 2010, 114, 12068-12079.

(37) Palmer, D. S.; Frolov, A. I.; Ratkova, E. L.; Fedorov, M. V. 749 Toward a Universal Model To Calculate the Solvation Thermody- 750 namics of Druglike Molecules: The Importance of New Experimental 751 Databases. Mol. Pharmaceutics 2011, 8, 1423-1429. 752

(38) Truchon, J.-F.; Pettitt, B. M.; Labute, P. A Cavity Corrected 3D- 753 RISM Functional for Accurate Solvation Free Energies. J. Chem. 754 Theory Comput. 2014, 10, 934-941.

(39) Palmer, D. S.; Misin, M.; Fedorov, M. V.; Llinas, A. Fast and 756 General Method to Predict the Physico-Chemical Properties of 757 Druglike Molecules using the Integral Equation Theory of Molecular 758 Liquids. Mol. Pharmaceutics 2015, 12, 3420.

759

(40) Jeanmairet, G.; Levesque, M.; Sergiievskyi, V.; Borgis, D. 760 Molecular Density Functional Theory for Water with Liquid-Gas 761 Coexistence and Correct Pressure. J. Chem. Phys. 2015, 142, 154112. 762 (41) Sergiievskyi, V.; Jeanmairet, G.; Levesque, M.; Borgis, D. 763 Solvation Free-Energy Pressure Corrections in the Three Dimensional 764 Reference Interaction Site Model. J. Chem. Phys. 2015, 143, 184116. 765 (42) Sergiievskyi, V.; Jeanmairet, G.; Levesque, M.; Borgis, D. 766 Pressure Correction in Classical Density Functional Theory: Hyper 767 Netted Chain and Hard Sphere Bridge Functionals. ArXiv150901409 768 Cond-Mat, 2015.

(43) Sergiievskyi, V. P.; Jeanmairet, G.; Levesque, M.; Borgis, D. Fast 770 Computation of Solvation Free Energies with Molecular Density 771 Functional Theory: Thermodynamic-Ensemble Partial Molar Volume 772 Corrections. J. Phys. Chem. Lett. 2014, 5, 1935-1942. 773

(44) Li, B.; Matveev, A. V.; Rösch, N. Three-Dimensional Reference 774 Interaction Site Model Solvent Combined with a Quantum 775 Mechanical Treatment of the Solute. Comput. Theor. Chem. 2015, 776 1070, 143-151.

(45) Krishtalik, L. I.; Alpatova, N. M.; Ovsyannikova, E. V. 778 Determination of the Surface Potentials of Solvents. J. Electroanal. 779 Chem. 1992, 329, 1-8.

780

(46) Fawcett, W. R. The Ionic Work Function and its Role in 781 Estimating Absolute Electrode Potentials. Langmuir 2008, 24, 9868- 782 9875.

(47) Netz, R. R.; Horinek, D. Progress in Modeling of Ion Effects at 784 the Vapor/Water Interface. Annu. Rev. Phys. Chem. 2012, 63, 401-418. 785

(48) Harder, E.; Roux, B. On the Origin of the Electrostatic Potential 786 Difference at a Liquid-Vacuum Interface. J. Chem. Phys. 2008, 129, 787 234706.

(49) Marenich, A.; Kelly, C.; Thompson, J.; Hawkins, G.; Chambers, 789 C.; Giesen, D.; Winget, P.; Cramer, C.; Truhlar, D. Minnesota Solvation 790 Database, version 2012; University of Minnesota: Minneapolis, 2012. 791 (50) Kelly, C. P.; Cramer, C. J.; Truhlar, D. G. Aqueous Solvation 792 Free Energies of Ions and Ion-Water Clusters Based on an Accurate 793 Value for the Absolute Aqueous Solvation Free Energy of the Proton. 794 J. Phys. Chem. B 2006, 110, 16066-16081. 795

(51) Tissandier, M. D.; Cowen, K. A.; Feng, W. Y.; Gundlach, E.; 796 Cohen, M. H.; Earhart, A. D.; Coe, J. V.; Tuttle, T. R. The Proton's 797 Absolute Aqueous Enthalpy and Gibbs Free Energy of Solvation from 798 Cluster-Ion Solvation Data. J. Phys. Chem. A 1998, 102, 7787-7794. 799 
800 (52) Beck, T. L. The Influence of Water Interfacial Potentials on Ion 801 Hydration in Bulk Water and near Interfaces. Chem. Phys. Lett. 2013, $802561-562,1-13$.

803 (53) Asthagiri, D.; Pratt, L. R.; Ashbaugh, H. S. Absolute Hydration 804 Free Energies of Ions, Ion-Water Clusters, and Quasichemical Theory. 805 J. Chem. Phys. 2003, 119, 2702-2708.

806 (54) Pollard, T. P.; Beck, T. L. The Thermodynamics of Proton 807 Hydration and the Electrochemical Surface Potential of Water. J. 808 Chem. Phys. 2014, 141, 18C512.

809 (55) Åqvist, J.; Hansson, T. Analysis of Electrostatic Potential 810 Truncation Schemes in Simulations of Polar Solvents. J. Phys. Chem. B 811 1998, 102, 3837-3840.

812 (56) Ashbaugh, H. S.; Sakane, S.; Wood, R. H. Reply to Comment on 813 "Electrostatic Potentials and Free Energies of Solvation of Polar and 814 Charged Molecules. J. Phys. Chem. B 1998, 102, 3844-3845.

815 (57) Vorobjev, Y. N.; Hermans, J. A Critical Analysis of Methods of 816 Calculation of a Potential in Simulated Polar Liquids: Strong 817 Arguments in Favor of "Molecule-Based" Summation and of Vacuum 818 Boundary Conditions in Ewald Summation. J. Phys. Chem. B 1999, 819 103, 10234-10242.

820 (58) Yang, P.-K.; Lim, C. Nonconvergence of the Solute Potential in 821 an Infinite Solvent and Its Implications in Continuum Models\|. J. 822 Phys. Chem. B 2002, 106, 12093-12096.

823 (59) Fedorov, M. V.; Kornyshev, A. A. Unravelling the Solvent 824 Response to Neutral and Charged Solutes. Mol. Phys. 2007, 105, 1-16. 825 (60) Genheden, S.; Luchko, T.; Gusarov, S.; Kovalenko, A.; Ryde, U. 826 An MM/3D-RISM Approach for Ligand Binding Affinities. J. Phys. 827 Chem. B 2010, 114, 8505-8516.

828 (61) Hünenberger, P. H.; McCammon, J. A. Effect of Artificial 829 Periodicity in Simulations of Biomolecules Under Ewald Boundary 830 Conditions: a Continuum Electrostatics Study. Biophys. Chem. 1999, $83178,69-88$.

832 (62) Hünenberger, P. H.; McCammon, J. A. Ewald Artifacts in 833 Computer Simulations of Ionic Solvation and Ion-Ion Interaction: A 834 Continuum Electrostatics Study. J. Chem. Phys. 1999, 110, 1856-1872. 835 (63) Rocklin, G. J.; Mobley, D. L.; Dill, K. A.; Hünenberger, P. H. 836 Calculating the Binding Free Energies of Charged Species Based on 837 Explicit-Solvent Simulations Employing Lattice-Sum Methods: An 838 Accurate Correction Scheme for Electrostatic Finite-Size Effects. J. 839 Chem. Phys. 2013, 139, 184103.

840 (64) Reif, M. M.; Hünenberger, P. H.; Oostenbrink, C. New 841 Interaction Parameters for Charged Amino Acid Side Chains in the 842 GROMOS Force Field. J. Chem. Theory Comput. 2012, 8, 3705-3723. 843 (65) Hummer, G.; Pratt, L. R.; García, A. E. Free Energy of Ionic 844 Hydration. J. Phys. Chem. 1996, 100, 1206-1215.

845 (66) Ekimoto, T.; Matubayasi, N.; Ikeguchi, M. Finite-Size Effect on 846 the Charging Free Energy of Protein in Explicit Solvent. J. Chem. 847 Theory Comput. 2015, 11, 215-223.

848 (67) Case, D. A.; Babin, V.; Berryman, J.; Betz, R.; Cai, Q.; Cerutti, 849 D.; T, C.III; Darden, T.; Duke, R.; Gohlke, H. et al. AMBER 14; 850 University of California: San Francisco, CA, 2014.

851 (68) Banks, J. L.; Beard, H. S.; Cao, Y.; Cho, A. E.; Damm, W.; Farid, 852 R.; Felts, A. K.; Halgren, T. A.; Mainz, D. T.; Maple, J. R.; et al. 853 Integrated Modeling Program, Applied Chemical Theory (IMPACT). 854 J. Comput. Chem. 2005, 26, 1752-1780.

855 (69) Marenich, A. V.; Jerome, S. V.; Cramer, C. J.; Truhlar, D. G. 856 Charge Model 5: An Extension of Hirshfeld Population Analysis for 857 the Accurate Description of Molecular Interactions in Gaseous and 858 Condensed Phases. J. Chem. Theory Comput. 2012, 8, 527-541.

859 (70) Maestro; Schrdinger, LLC.: New York, 2014.

860 (71) In Computer Simulation of Liquids; Allen, M. P., Tildesley, D. J., 861 Eds.; Clarendon Press: Oxford, UK, 1987.

862 (72) Frisch, M. J.; Trucks, G. W.; Schlegel, H. B.; Scuseria, G. E.; 863 Robb, M. A.; Cheeseman, J. R.; Scalmani, G.; Barone, V.; Mennucci, 864 B.; Petersson, G. A. et al. Gaussian 09, Revision D.01; Gaussian, Inc.: 865 Wallingford, CT, 2009.

866 (73) Zhao, Y.; Truhlar, D. G. The M06 Suite of Density Functionals 867 for Main Group Thermochemistry, Thermochemical Kinetics, Non868 covalent Interactions, Excited States, and Transition Elements: Two
New Functionals and Systematic Testing of Four M06-Class 869 Functionals and 12 Other Functionals. Theor. Chem. Acc. 2008, 120, 870 215-241.

871

(74) Lynch, B. J.; Zhao, Y.; Truhlar, D. G. Effectiveness of Diffuse 872 Basis Functions for Calculating Relative Energies by Density 873 Functional Theory. J. Phys. Chem. A 2003, 107, 1384-1388. 874

(75) Marenich, A.; Cramer, C.; Truhlar, D. CMSPAC; University of 875 Minnesota: Minneapolis, MN, 2013.

876

(76) Pronk, S.; Páll, S.; Schulz, R.; Larsson, P.; Bjelkmar, P.; 877 Apostolov, R.; Shirts, M. R.; Smith, J. C.; Kasson, P. M.; van der Spoel, 878 D.; et al. GROMACS 4.5: a High-Throughput and Highly Parallel 879 Open Source Molecular Simulation Toolkit. Bioinformatics 2013, 29, 880 845-854.

881

(77) Berendsen, H. J. C.; Grigera, J. R.; Straatsma, T. P. The Missing 882 Term in Effective Pair Potentials. J. Phys. Chem. 1987, 91, 6269-6271. 883

(78) Hansen, N.; van Gunsteren, W. F. Practical Aspects of Free- 884 Energy Calculations: A Review. J. Chem. Theory Comput. 2014, 10, 885 2632-2647.

(79) Darden, T.; York, D.; Pedersen, L. Particle Mesh Ewald: An N. 887 $\log (\mathrm{N})$ Method for Ewald Sums in Large Systems. J. Chem. Phys. 1993, 888 98, 10089-10092.

(80) Berendsen, H. J. C.; Postma, J. P. M.; van Gunsteren, W. F.; 890 DiNola, A.; Haak, J. R. Molecular Dynamics with Coupling to an 891 External Bath. J. Chem. Phys. 1984, 81, 3684-3690.

(81) Luchko, T.; Gusarov, S.; Roe, D. R.; Simmerling, C.; Case, D. 893 A.; Tuszynski, J.; Kovalenko, A. Three-Dmensional Molecular Theory 894 of Solvation Coupled with Molecular Dynamics in Amber. J. Chem. 895 Theory Comput. 2010, 6, 607-624.

(82) Perkyns, J.; Pettitt, B. M. A site-site Theory for Finite 897 Concentration Saline Solutions. J. Chem. Phys. 1992, 97, 7656-7666. 898

(83) Kovalenko, A.; Hirata, F. Potentials of Mean Force of Simple 899 Ions in Ambient Aqueous Solution. I. Three-Dimensional Reference 900 Interaction Site Model Approach. J. Chem. Phys. 2000, 112, 10391- 901 10402.

(84) Kleine, J. D.; Johansson, J.; Yamazaki, T.; Gusarov, S.; 903 Kovalenko, A.; Case, D. A.; Luchko, T. Explicit Solvent Host-Guest 904 Binding Free Energy Calculations; http://www.csun.edu/sites/ 905 default/files/Luchko,\%20Tyler_0.pdf (accessed January 21, 2016). 906

(85) Miyata, T.; Hirata, F. Combination of Molecular Dynamics 907 Method and 3D-RISM Theory for Conformational Sampling of Large 908 Flexible Molecules in Solution. J. Comput. Chem. 2008, 29, 871-882. 909

(86) Casanova, D.; Gusarov, S.; Kovalenko, A.; Ziegler, T. Evaluation 910 of the SCF Combination of KS-DFT and 3D-RISM-KH; Solvation 911 Effect on Conformational Equilibria, Tautomerization Energies, and 912 Activation Barriers. J. Chem. Theory Comput. 2007, 3, 458-476. 913

(87) Kloss, T.; Heil, J.; Kast, S. M. Quantum Chemistry in Solution 914 by Combining 3D Integral Equation Theory with a Cluster 915 Embedding Approach. J. Phys. Chem. B 2008, 112, 4337-4343. 916

(88) Cramer, C. J.; Truhlar, D. G. A Universal Approach to Solvation 917 Modeling. Acc. Chem. Res. 2008, 41, 760-768. 